\title{
Classification of Primary Brain Tumors: Molecular Aspects
}

\author{
Péter Molnár \\ University of Debrecen, Medical and Health Sciences Center, Department of Pathology \\ Hungary
}

\section{Introduction}

The guiding principle of this chapter is the importance of combining traditional morphological data with the recently acquired knowledge on the genetic and epigenetic signalling pathways which drive the evolution of CNS tumors. The official World Health Organization terminology (WHO, 2007) will be used since it is the source of information for neurooncology, neuroradiology, neurosurgery, etc. It is of outmost importance that new entities have emerged since 2007 and it seems inevitable to see a new classification in the near future. It is beyond the scope if this chapter to dwell on the pitfalls in diagnostic surgical neuropathology, suffice is to say that the classic, traditional case descriptions which are usually presented in textbooks comprise only a fraction of diagnostic dilemmas so often hunting practicing neuro-oncopathologists.

The main focus will be placed on intracranial lesions but it needs to be emphasized that the diagnostic entities which come the way of a surgical neuropathologist broadly overlap with an extensive range of entities which basically belong to hematopathology, soft tissue and bone/chondroid tumors.

In all textbooks on neuropathology the usual way to start is to point out that knowledge of brain and spinal cord anatomy as well as familiarity with the broad range of cellular reactions in diseased CNS are essential for success in understanding pathology reports. There is no way to cover all these details, hence a list of reference books is provided. This chapter is born out of the need to outline unambiguous and consistent terminology of brain tumors. This together with a strong urge to define the use of a universally accepted grading system (be that weak as it is despite of century-old efforts) could help not only all specialists who are involved in treating brain tumor patients but also and of outmost importance the patients themselves.

\section{Pathology of brain tumors - A general overview}

Brain tumors are not that common (about 1-1.5\% of neoplasms in adults) but they still present an often frustrating challenge for oncologists even in developed countries. In contrast to the somewhat limited pathologic variations of adult brain tumors the extreme phenotypic diversity of pediatric brain tumors often results in overwhelming diagnostic problems even for the experienced pediatric pathologist. A recent report from France states that over 5 years, 25756 cases of newly diagnosed and histologically confirmed primary 
CNS tumors (PCNST) have been recorded. Histological diagnoses included glioma 48.9\%, all other neuroepithelial tumors 5\%, meningioma $28.8 \%$, nerve sheath tumor $8.4 \%$, lymphoma $3.2 \%$ and others $5.7 \%$.

Epidemiological data on the most common intracranial tumors are presented in Table 1 . The data are from the 2011 CBTRUS Statistical Report (http://www.cbtrus.org/2007-2008/200720081.html.)

\begin{tabular}{|lcccc|}
\hline \multicolumn{1}{|c}{ Tumor type } & Grade & Incidence & Male/Female & $\begin{array}{c}\text { 5-year- } \\
\text { survival (\%)c }\end{array}$ \\
\hline $\begin{array}{l}\text { Pilocytic } \\
\text { astrocytoma }\end{array}$ & I & 0.27 & 1.0 & 94 \\
$\begin{array}{l}\text { Diffuse astrocytoma } \\
\text { Anaplastic }\end{array}$ & II & 0.15 & 1.2 & 48 \\
$\begin{array}{l}\text { astrocytoma } \\
\text { Glioblastoma }\end{array}$ & III & 0.48 & 1.4 & 27 \\
Oligodendroglioma & II/III & 0.38 & 1.6 & 5 \\
Ependymoma & II/III & 0.24 & 1.5 & 80 \\
Medulloblastoma & IV & 0.26 & 1.4 & 62 \\
Meningeoma & I (II/III) & 0.75 & 1.6 & 67 \\
\hline
\end{tabular}

a: WHO 2007; b: New case/1 million population/year (1995-2007, CBTRUS; USA); c: observed chance of survival compared to the expected life span (,survival") of the reference population (USA). SEER (Survival, Epidemiology and End Results) 2010. d. The five-year-long survival of anaplastic oligodendrogliomata is $49 \%$. e. Intracranial and spinal meningeomata combined.

Table 1.

The direct cause of brain tumors is largely unknown. Inherited tumor-syndromes have helped to shed light on various genetic abnormalities which might play a role in tumorinduction. It is well documented that radiation of the head-and-neck region is associated with an increasing number of meningiomas. Primary CNS lymphomas (PCNSLs) are the most common causes of non-infective intracranial space occupying lesions in immunocompromised people. The frequency of PCNSL has increased almost by an order of a magnitude (3-10x) and this tendency is present among those with normal immune system. An increased incidence of gliomas has become obvious by the last decade of the 20th century, and this is not due to the advanced sensitivity of diagnostic tools.

Although in most cases the etiology of neoplastic transformation awaits clarification there has been a truly revolutionary explosion of genetic and molecular tools giving new information that has fundamentally changed both basic and translational (clinical) neurooncology. Analysis of genetic instability and basic data on invasion and angiogenesis have all became inescapable questions to pursue during routine diagnostic activity. Information on gene expression profiles, application of tissue microarray techniques, follow up on 
mRNA splicing patterns, are now considered to be part of the state-of-the-art workup in brain tumor analysis. These inevitably depend on functional genomics and bioinformatics. It is fair to say that the last 2-3 years have fundamentally changed our concept of diagnostic, prognostic and predictive markers in neuro-oncology.

All these changes require a new attitude towards tissue samples: the neurosurgeon must be aware of the importance of sampling that will critically influence the pathologist's ability to outline the major aspects of tailored therapy. Hence it cannot be overemphasized that the decisions on the distribution of various parts of a biopsy between diagnostic labs and research areas (including tumor banks) are the sole and outmost responsibility of the pathologist. This is the only way to ensure that the Hippocratic requirement of "Salus aegroti suprema lex esto ${ }^{1}$ " is realized. No academic goal may interfere with the proper, individual, personalized diagnosis! The precise diagnosis (both radiological and pathological) of brain tumors is significantly dependent on the fact that certain entities are linked with specific geographical areas (anatomical locations) within the CNS. Similarly, age does have an almost pathognomonic relevance in most cases. As always, exceptions to this fundamental rule are not infrequent, still it is the combined knowledge of radiological characteristics, exact site of the lesion and the patient's age which is an indispensible guidance for the pathologist.

\subsection{Histological classification of various tumor types affecting the central nervous system}

It is mentioned above that the last WHO classification was published in 2007. Since then new entities have been recognized. The idea that the pattern of differentiation is reflected by phenotypic features (in other words, phenotype may give a clue about the cell of origin) has been retained in the WHO classification. Despite of a long-lasting dispute it has not been fully decided whether "retrogressive" differentiation (loss of maturity from a mature state) can or cannot result in cancer formation. Meanwhile it has become generally accepted that tumors most often arise from "cancer stem cells". The term tumor initiating cells (TICs) probably describes best the features of these progenitor elements which are assumed to be capable of self-renewal and of divergent differentiation. It is worth noting here that these "stem cells" are hypothesized to strongly interact with their immediate micromilieau (niche). As a result the actual TICs' behavior and chemo- and/or radioresistance will be modified by the niche elements (endothelial cells, microglia, concentration of various signalling molecules, etc.). This explains why these cells which are often of low proliferative activity can maintain tumor growth or recurrence after vigorous treatment.

Table 2. is construed basically on the WHO 2007 classification with some modifications. It is important for oncologists to be aware of new entities which most likely will be included in the next WHO book and already occur in pathology reports. It must repeatedly be stressed that the frequency, location and types of CNS tumors vary with age and to some degree with sex. The largest group of tumors comprises neuroepithelial neoplasms, a significant portion of intracranial tumors are meningeal and metastatic lesions. Gliomas (astrocytomas, oligodendrogliomas and ependymomas) account for circa $40 \%$ of all tumors and approximately $80 \%$ of malignant tumors. The most comprehensive and up-to-date information on the epidemiology of various CNS tumors is available on the CBTRUS website shown above.

${ }^{1}$ In a rather free translation: „Everything is to serve the best interest of the patient.” 


\section{Neuroepithelial tumors}

a. Astrocytic tumors

Pilocytic astrocytoma

Pilomyxoid astrocytoma

Pleomorphic xanthoastrocytoma

Diffuse astrocytomas

Anaplastic astrocytoma

Glioblastoma

Glioblastoma with significant oligodendroglial component (GBO)

Giant cell glioblastoma

Gliosarcoma

Gliomatosis cerebri

b. Oligodendroglial tumors

Oligodendroglioma

Anaplastic oligodendroglioma

Oligoastrocytoma

Anaplastic oligoastrocytoma

c. Ependymal tumors

Ependymoma

Anaplastic ependymoma

Myxopapillary ependymoma

Subependymoma

d. Choroid plexus tumors

Choroid plexus papilloma

Atypical choroid plexus papilloma

Choroid plexus carcinoma

e. Other neuroepithelial tumors

Astroblastoma

Chordoid glioma of the third ventricle

Angiocentric glioma

II. Neuronal and mixed neuronal-glial tumors

a. Neuronal tumors

Gangliocytoma and ganglioglioma

Dysplastic gangliocytoma of the cerebellum

Central neurocytoma and variants

b. Mixed neuronal-glial tumors

Ganglioglioma

Anaplastic ganglioglioma

Desmoplastic infantile astrocytoma and ganglioglioma (DIA/DIG)

Central neurocytoma and extraventricular neurocytoma

Cerebellar liponeurocytoma

Papillary glioneural tumor (PGNT)

Rosette-forming glioneural tumor of the $4^{\text {th }}$ ventricle (RGNT)

Paraganglioma (spinal)

Dysembryoplastic neuroepithelial tumor 


\section{Tumors of the pineal region}

Pineocytoma

Pineal parenchymal tumor of intermediate differentiation

Pineoblastoma

Papillary tumor of the pineal region

\section{Tumors of the sellar region}

Pituitary adenoma

Craniopharyngeoma

Granular cell tumor of the neurohypophysis

Pituicytoma

Spindle cell oncocytoma of the adenohypophysis

\section{Embryonal tumors}

Medulloblastoma

Classical medulloblastoma

Desmoplastic/nodular medulloblastoma

Medullomyoblastoma

Melanotic medulloblastoma

CNS primitive neuroectodermal tumor (cPNET)

Medulloepithelioma

Ependymoblastoma

Atypical rhabdoid/teratoid tumor (AT/RT)

\section{Tumors of the cranial nerves}

Schwannoma (Neurilemmoma)

Neurofibroma

Perineurioma

Malignant peripheral nerve sheath tumor (MPNST)

\section{Meningeal tumors}

Meningiomas

Mesenchymal, non-meningothelial tumors

Hemangiopericytoma

Melanotic lesions

Hemangioblastoma

VIII. Tumors of the hemopoietic system

Malignant lymphomas

Histiocytic tumors

\section{Germ cell tumors}

CNS germ cell tumors

\section{$X$. Familial tumor syndromes}

Neurofibromatosis type I. (NF1)

Neurofibromatosis type II. (NF2)

Schwannomatosis 
von Hippel-Lindau disease and haemangioblastoma

Tuberous sclerosis complex and subependymal giant cell astrocytoma (SEGA)

Lhermitte-Duclos disease

Cowden disease and dysplastic gangliocytoma of the cerebellum

Turcot syndrome

Li-Fraumeni syndrome and TP53 germline mutations

Rhabdoid tumor predisposition syndrome

Naevoid basal cell carcinoma syndrome

\section{Metastatic tumors of the CNS}

Table 2.

The various names date back to the first comprehensive classification of nervous system tumors put together by Percival Bailey and Harvey Cushing in 1926. This was based on presumed parallels between embryologic cell forms and neoplastic elements. This nomenclature is still in use, however much of the terminology has changed fundamentally.

Neoplasia is a genetic disease and now there is abundant information at hand about alterations which occur in key regulatory genes, either inherited in mutant forms or arising sporadically as a result of somatic noxae to growth regulatory genes. Tumors may come about when the function of protein products of key growth regulatory genes is altered or they can develop as a result of random genetic changes which are not properly corrected by DNA repair mechanisms. As it is shown in Fig. 1. current hypotheses drastically overwrite our previous concepts about the origin of neoplastic cells. It has for a long time been held that terminally differentiated cells are incapable to form neoplasms. Recent observations indicate that non-canonical steps, like epithelial-mesenchymal transformation (EMT) or the reverse (MET) may be involved in tumorigenesis and metastatic spread of cancer. In light of these data the hypothetical routes (indicated by dashed lines in the Figure 1) of regressive differentiation become easier to comprehend. This scenario is being actively researched and doubtlessly important role is allocated to cells with pluripotency in the evolution of brain tumors. NB. These cells are often referred to as tumor stem cells, although they do not necessarily fulfill the requirements of bona fide stem cells. Figure 1 also shows that there is no common understanding of how ependymal tumors fit into this hypothesis, neither it is clear how exactly mixed glial tumors evolve. The latter phenomenon might be related to clonal selection during the accumulation of genetic damage as tumors progress. In this biological, pathological sense tumor progression is strictly related to dedifferentiation (i. e., increasing grade) and not to the change of total tumor volume, as it is often used in clinical, particularly radiological terminology. Cellular heterogeneity and the almost inevitable progression (accumulation of genetic abnormalities inducing dedifferentiation) are paramount intrinsic features of glial tumors.

The detailed description of histological and cellular features of individual tumor types defies the length of this chapter. Moreover, neither neurosurgeons, nor neuro-oncologists are benefiting from these textbook data. However, if an individual tumor carries unusual features and still needs to be squeezed in an existing category (mainly because health insurance policies often require traditionally "boxed" entities), it is the responsibility of the MDT to come to an agreement about a category which is suitable to fit in treatment protocols. Furthermore, descriptive analysis is bound to be enriched by individualized genetic and molecular data which eventually might carry more relevant information vis-ávis treatment than the classical histo- and cytopathologic characteristics (vide infra). 


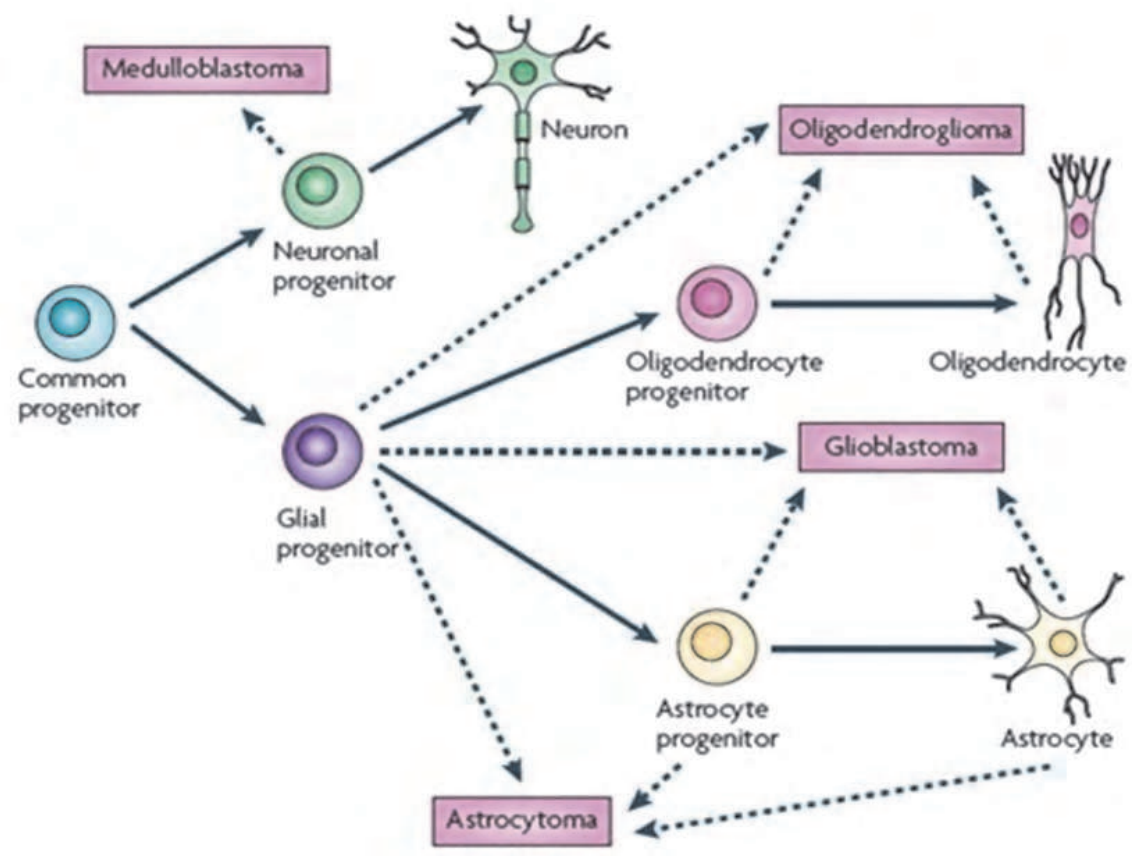

Fig. 1. Origin of neuroglial tumors. Modified after Huse JT and Holland EC. Nature Reviews Cancer doi:10.1038/nrc2818.

\subsection{Grading of CNS tumors}

The WHO classification grades tumors according to their malignant potential on a scale ranging from 1 for "benign" tumors to 4 for the most aggressive and malignant ones. Other grading systems have been used in the past, but the WHO 2007 system is now the one which is the most easy to find and is the one best recognized by pathologists, radiologists, neurosurgeons and oncologists. Modifications of the Kernohan system of grading were adopted by the WHO ever since the publication of the first "blue book" (1979). It was first revised in 1993, then in 2000 and 2007. It is very important to keep in mind that grading criteria are always artificial, arbitrary and despite of all world-wide efforts to avoid it, are always very subjective. Various techniques of the ever growing field of molecular diagnostics are increasingly employed not only in tumor classification but also in tumor grading. At this day and age it is still prudent to state that morphologic findings endure as a golden standard for proper evaluation of genetic and molecular observations.

The original concept of grading was introduced by Broders who first realized that the degree of cellular differentiation does have a reliable value in predicting the biological behavior of cancerous growths of the lip. He hypothesized that the "cell-of-origin" model reflects clinical prognosis: if more than $75 \%$ of the cells are "like" the cell of origin (i. e., stratified squamous epithelium) indicating that the neoplastic elements retain significant degree of differentiation then the outlook is favorable. Since it proved to work well for a host of systemic cancers Kernohan introduced the idea to brain tumor analysis and suggested a four-tier grading model. 


\begin{tabular}{|l|l|l|}
\hline \multicolumn{3}{|c|}{ Four-tier grading scheme } \\
\hline Grade 1 & Low proliferative potential and possibility of surgical cure & $\begin{array}{l}\text { Well- } \\
\text { differentiated }\end{array}$ \\
\hline Grade 2 & Diffuse growth, tendency to recur, though only few mitoses & $\begin{array}{l}\text { Moderately- } \\
\text { differentiated }\end{array}$ \\
\hline Grade 3 & $\begin{array}{l}\text { Histological evidence of malignancy, destructive growth, brisk } \\
\text { mitotic activity, anisonucleosis }\end{array}$ & $\begin{array}{l}\text { Poorly- } \\
\text { differentiated }\end{array}$ \\
\hline Grade 4 & Cytologically malignant, mitotically active, necrosis-prone & Anaplastic \\
\hline
\end{tabular}

Eventually it has become increasingly obvious that the 4-tier-grading can be applied only with significant difficulties even to astrocytic tumors. Ringertz then applied a 3-tiered scheme for astrocytomas.

\begin{tabular}{|l|l|l|}
\hline \multicolumn{2}{|c|}{ Three-tier grading scheme } \\
\hline Grade 1 & Low grade & Well-differentiated \\
\hline Grade 2 & Intermediate grade & Moderately differentiated \\
\hline Grade 3 & High grade & Poorly-differentiated \\
\hline
\end{tabular}

This was replaced by the St.Anne/Mayo classification which was doomed because of the inclusion of a rather elusive and rarely occurring "Grade I." lesion: diffuse astrocytic tumors without atypia. The current concept holds that Grade I. astrocytomas are relatively circumscribed (sic!), while all other grades by definition comprise diffusely growing tumors. It is obvious from the 2007 WHO classification that certain tumors have only 3 grades, however, the "missing" $4^{\text {th }}$ category may be at the origo of the scale (ependymal, oligodendroglial, or mixed glial tumors) and thus the highest (most malignant) grade is Gr. III., which implies that a Gr. III. oligoastrocytoma (OA Gr. III.) often presents all cytological and histological features of the most malignant (Gr. IV.) astrocytoma, i. e., glioblastoma (GBM). Needless to say this often results in confusion, particularly for the oncologist whose decision on therapy is often highly determined by bureaucratic regulations (e. g., financial limitations of treatment options). To complicate issues further several tumors do not have Grade I. forms at all (oligoastrocytic tumors, OAs), while some Gr. I. lesions are strictly related to anatomic location (myxopapillary ependymoma or subependymal giant cell astrocytoma [SEGA]). A firm understanding of the meaning and significance of numerical grading among all people (usually members of the MDT $=$ multidisciplinary team) is quintessential, particularly since the criteria used for assigning diagnoses and grades are different between the various systems in use, sometimes even between the older and newer WHO classifications. Numerical grades do not surmount the need for a fundamental understanding of the histological/cytological criteria for diagnosis of specific types of tumors.

It is important to note that the definition of biological malignancy as reflected by increasing dedifferentiation is not necessary for lethal clinical outcome of a tumor. A highly differentiated meningeal tumor (Gr. I.) may kill a patient if it mechanically compresses vital, eloquent areas (meningioma of the edge of the foramen magnum). By the same token, the general principle of oncology that states that the most important indication of malignancy is metastatic spread does not hold in neuro-oncology. Even GBMs, which are considered to be the most malignant forms of gliomata rarely metastasize outside CNS compartments. 
Table 3. summarizes the currently defined WHO grades within the most common CNS tumors. As a general rule staging is not applicable to CNS tumors and grading of non-CNS specific tumors can be found in the proper volume of the WHO series as well as in the $7^{\text {th }}$ Edition of Cancer Staging Handbook. Neither Table 2. nor Table 3. go into details of various subtypes of primary and/or secondary CSN tumors. Everyone should keep in mind that chondrogenic, osteogenic, fibroblastic, etc. tumors may affect the brain or spinal cord either when arising de novo within the intracranial or intraspinal compartments or when they directly invade CNS tissue from adjacent structures. Their grading (and staging, for that matter) follows the general principles of systemic pathology. It is noteworthy, however, that the tight and non-compliant intracranial space poses additional biological/clinical problems in the latter cases: increased ic. pressure, brain herniation, etc. complicate treatment of these neoplasms.

\subsection{Limitations of morphological grading}

To demonstrate the subjectivity of glioma grading it is worth comparing the features of pilocytic tumors (as listed in the WHO fascicle) with those of GBMs. While doing so two definitions need to be taken into account. The highest glioma grade is assigned to tumors which have either endothelial proliferation and/or necrosis in the neoplasm. "Endothelial proliferation" nowadays is more often replaced by "MVP" (=microvascular proliferation). This phenomenon means not solely apparent multilayering of endothelial cells but a complex process that comprises endothelial atypia, endothelial mitotic activity (often accompanied by abnormal p53 immunohistochemical abnormalities, vide infra), and endothelial cell (EC) multiplication accompanied by an abnormal deposition of basal lamina (BL) components plus proliferative activity of pericytic elements. This often results in the formation of literally "glomerulum ${ }^{2 "}$-like elements (glomeruloid proliferation), but the latter is not a sine qua non of Gr. IV. Not infrequently these abnormal vascular structures occur along the edge of the infiltrative zone or partially surrounding necrotic areas ("vasocorona"). Necrosis is another histological feature which often indicates malignant potential. This is not necessarily an indication of the parenchyma's outgrowing the blood supply (as general pathology often states). Glial tumors are known to be very vascular but a heretofore unclarified complex dysregulation results in what is called "sui generis" tumor necrosis. The fundamental pathobiology of this phenomenon exceeds the limits of this chapter, it suffices to say that this necrosis (surrounded by palisading tumor cells) is fundamentally different from the "garden variety" infarctions. The latter are also common, since despite of rich neovascularization blood supply to gliomas is often interrupted by thromboses which may affect all sized vessels.

Daily experiences firmly indicate that there is a myth surrounding numerical grades particularly since the problem usually is presented in almost all textbooks in a way that suggests: criteria are strict, obvious, and "self-evident" and their use is only a matter of routine. The following paragraphs are intended to shed light on the complexity of the issue. First I will quote from the last WHO book those sentences which describe features of pilocytic tumors which are the prime examples of a grade I. tumor (WHO 2007, pp15-20). It is worth to think through these quotations keeping in mind what almost everybody considers as prima facie evidence of the worst grade, i. e., glioblastoma.

2 The "magic" capillary structure that occupies the Bowman space. 


\begin{tabular}{|c|c|c|c|c|c|c|c|c|c|}
\hline & \multicolumn{4}{|c|}{ Astrocyto tumours } & & 1 & " & w & N \\
\hline \multirow{2}{*}{$\begin{array}{l}\text { Subependymal giant cel } \\
\text { astrogyoma }\end{array}$} & \multirow[b]{2}{*}{$\cdot$} & & & & \multirow{2}{*}{$\begin{array}{l}\text { Central neurocyloma } \\
\text { Extraventrioular neurocyoma }\end{array}$} & & \multirow{2}{*}{$\dot{\cdot}$} & & \\
\hline & & & & & & & & & \\
\hline Pilocytic astrocytoma & $\cdot$ & & & & Cerebellar lponeurocyloma & & $\cdot$ & & \\
\hline Pilomyooid astrocytoma & & $\cdot$ & & & Paraganglioma of the spinal cord & $\cdot$ & & & \\
\hline Difuse astrocytoma & & $\cdot$ & & & Papilary glioneuronal tumour & $\cdot$ & & & \\
\hline Pleomorphic xanthoastrocytoma & & $\cdot$ & & & \multirow{2}{*}{$\begin{array}{l}\text { Rosette-forming glioneuronal } \\
\text { tumour of the fourth ventriclo }\end{array}$} & \multirow[b]{2}{*}{ • } & \multirow{2}{*}{ 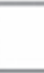 } & & \\
\hline Anaplastic astrocytoma & & & - & & & & & & \\
\hline Glioblastoma & & & & $\cdot$ & \multirow{2}{*}{\multicolumn{5}{|c|}{ Plnoal tumours }} \\
\hline Giant cell globlastoma & & & & $\cdot$ & & & & & \\
\hline Gliosarcoma & & & & $\cdot$ & Pineocyoma & $\cdot$ & & & \\
\hline \multicolumn{5}{|l|}{ Oligodendrogllal tumours } & $\begin{array}{l}\text { Pineal parenchymal tumour of } \\
\text { intermediate differentiation }\end{array}$ & & - & - & \\
\hline Olgodendroglioma & & $\cdot$ & & & Pineoblastoma & & & & $\cdot$ \\
\hline Anaplastic oligodendroglioma & & & - & & Papillary tumour of the pineal region & & $\cdot$ & $\cdot$ & \\
\hline \multicolumn{5}{|l|}{ Ollgoastrocyle tumours } & \multicolumn{5}{|l|}{ Embryonal tumours } \\
\hline Oligoastrocyloma & & $\cdot$ & & & Medulloblastoma & & & & - \\
\hline Anaplastic oligoastrocyloma & & & $\cdot$ & & CNS primitive neuroectodermal & & & & \\
\hline \multirow{2}{*}{\multicolumn{5}{|c|}{ Ependymal tumours }} & tumour (PNET) & & & & $\cdot$ \\
\hline & & & & & Atypical teratoid / thabdoid tumour & & & & $\cdot$ \\
\hline Subependymoma & $\cdot$ & & & & \multirow{2}{*}{\multicolumn{5}{|c|}{ Tumours of the cranial and paraspinal nerves }} \\
\hline Myxopapillary ependymorna & $\cdot$ & & & & & & & & \\
\hline Ependymoma & & $\cdot$ & & & Schwamoma & $\cdot$ & & & \\
\hline Anaplastic ependymoma & & & $\cdot$ & & Neurofioroma & $\cdot$ & & & \\
\hline \multirow{2}{*}{\multicolumn{5}{|c|}{ Chorold plexus tumours }} & Perinourioma & $\cdot$ & $\cdot$ & $\cdot$ & \\
\hline & & & & & \multirow{2}{*}{$\begin{array}{l}\text { Malignant peripheral nevve } \\
\text { sheath tumour (MPNST) }\end{array}$} & & \multirow[b]{2}{*}{$\cdot$} & & \\
\hline Choroid plexus papilloma & $\cdot$ & & & & & & & $\cdot$ & $\cdot$ \\
\hline Alypical choroid plexus papilloma & & - & & & & & & & \\
\hline Choroid plexus carcinoma & & & $\cdot$ & & Meningeal tumours & & & & \\
\hline & & & & & Meningioma & $\cdot$ & & & \\
\hline Other neuroepithellal tumours & & & & & Atypical meningioma & & $\cdot$ & & \\
\hline Angiocentric glioma & $\cdot$ & & & & Anaplastic / malignart meningioma & & & $\cdot$ & \\
\hline Chordoid giloma of & & & & & Haemangicpericyloma & & $\cdot$ & & \\
\hline the third vertencle & & $\cdot$ & & & Anaplastic haemangioperioytoma & & & $\cdot$ & \\
\hline & & & & & Haemangicblastoma & $\cdot$ & & & \\
\hline Neuronal and mbeed neuronal-glial t & mou & & & & & & & & \\
\hline Gangliocyioma & $\cdot$ & & & & Tumours of the sellar region & & & & \\
\hline Ganglioglioma & $\cdot$ & & & & Craniophanyoloma & $\cdot$ & & & \\
\hline Anaplastic ganglioglioma & & & $\cdot$ & & Granular cel tumour & & & & \\
\hline Desmoplastic intantile astrocyoma & & & & & of the neurohypophysis & • & & & \\
\hline and ganglioglioma & $\cdot$ & & & & Pituicyloma & $\cdot$ & & & \\
\hline $\begin{array}{l}\text { Dysembryoplastic } \\
\text { neurcepithelial tumour }\end{array}$ & - & & & & $\begin{array}{l}\text { Spindle cell onoocynoma } \\
\text { of the adenohypophysis }\end{array}$ & • & & & \\
\hline
\end{tabular}


"...hyperchromatic and pleomorphic nuclei, glomeruloid vascular proliferation, infarct-like necrosis and infiltration of leptomeninges are compatible with the diagnosis of pilocytic astrocytoma"; "....degenerative atypia with pleomorphism, smudgy chromatin, and nuclear-cytoplasmic pseudoinclusions (are) frequently seen in long-standing lesions"; "enfilades resemble those of what was termed the 'primitive polar spongioblastoma' (may occur)"; "one study found the acquisition of atypia, particularly of increased cellularity and occasional mitoses, to be of no prognostic significance". - It is also noted that pilocytic astrocytoma may undergo malignant transformation: "They often feature multiple mitoses per single high power field, endothelial proliferation and palisading necrosis. Such tumors should not be designated glioblastoma..." - given all these statements from the "horse's mouth" (i. e., the most authoritative book on CNS tumors) it is not surprising that unless there is a firm understanding of the complexity of issues at hand oncologists often are dumbfounded when having read the descriptive part of a report they expect the worst (Gr. IV.) still they are given the best (Gr. I.) possible diagnosis. How is that possible?

One should keep in mind that morphological evaluation is not a black-or-white type of decision forming intellectual activity; it is an analogous thinking process. Pathologists compare a waste amount (in fortunate situations) of mentally stored images to the actual fields in the microscope, they compare images-to-images, complex patterns to complex patterns and then put everything in context (age, sex, imaging features, intraoperative findings, macroscopic features, etc.) - provided they do have all this information. Anyone familiar with the image below can decide how objective and reliable analogous thinking is:

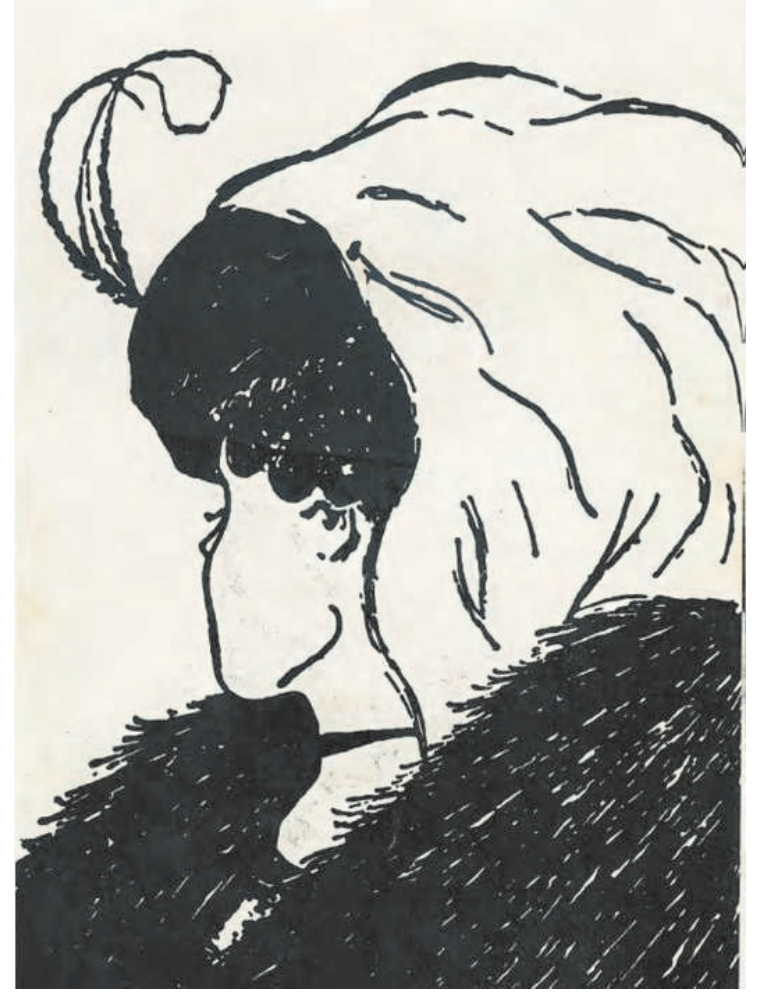


The dilemma is more obvious if one considers the proverbial pathologist's answer to the clinicians' question: Why is the diagnosis "this" and not "that"? - Because it looks more like "this" than "that"! And it does not mean that the pathologist is incompetent, the problem is inherent in the psychophysiology of "analogous thinking". There is nothing else that would emphasize MDT work's importance more than realization of this theoretical problem.

To drop another stone into the seemingly quiet pond: there are physical limitations as well, namely, sampling errors. This is demonstrated (in an analogous way) with the help of the following images, which show two sides of the same apple from my kitchen table:

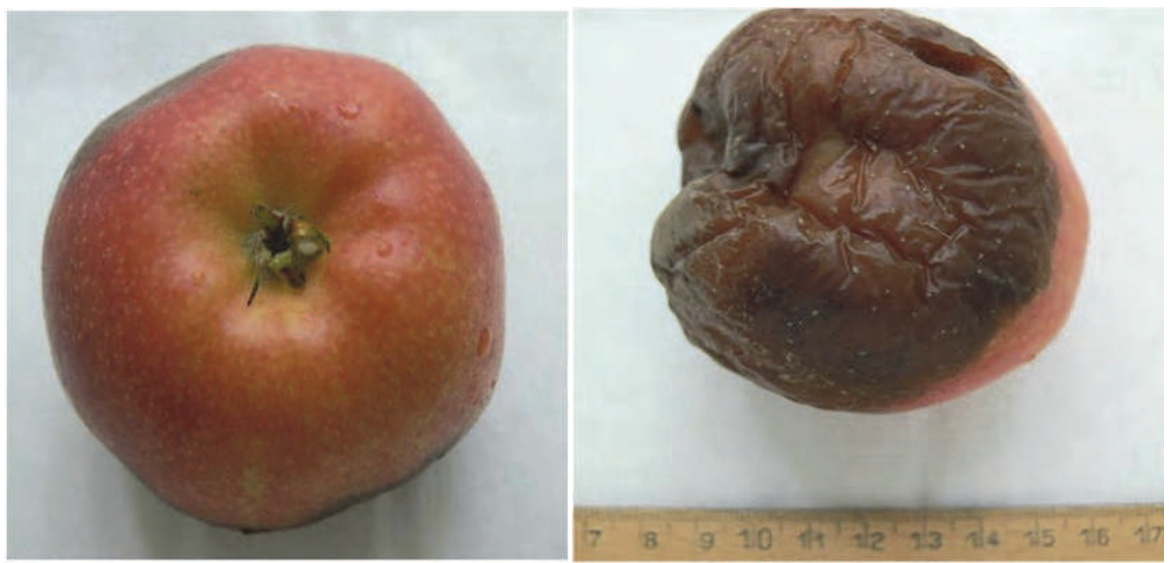

Obviously often the pathologist does not have a chance to evaluate the whole lesion, in 3D, from all aspects. He is absolutely dependent upon the guidance of the neuroradiologist (who may be able to grade tumours in vivo [see Jakab et al]) and the neurosurgeon. Unfortunately the latter's freedom of sampling is often highly limited and that complicates issues further:

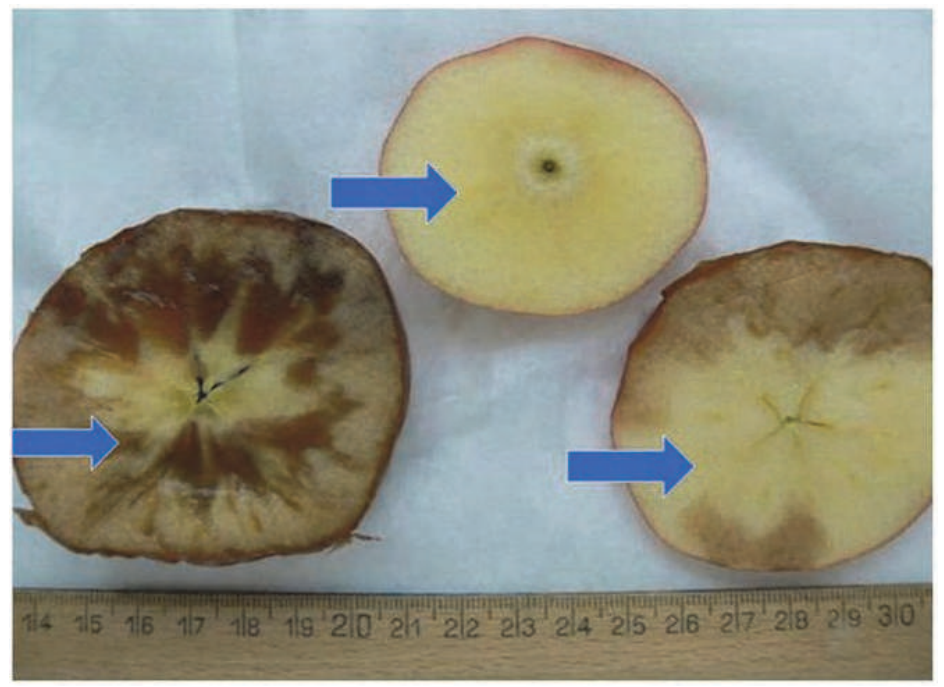


The blue arrows indicate the variability and often non-representative nature of sampling. Imagine the inherent errors in grading when comparing the 3 biopsies from the same apple ("tumor"). Added to the problem is the almost pathognomonic regional heterogeneity of gliomas, particularly of high grade astrocytic tumors. Any neuro-oncopathologist who regularly diagnoses glioma samples could without any specific effort take a series of microphotographs from a GBM demonstrating tumor grades from I through IV.

So, what is the conclusion? Can anyone trust pathologists for any degree of reliability? The answer is yes, but with adjuncts. Unless one is asking for legal and/or ethical problems should only give an opinion only if she or he is convinced that all necessary information is provided plus no part of the tissue has been retained (for scientific reasons or tissue banking). It is the prerogative of the pathologist to select the areas which are eventually embedded, sectioned, stained and analyzed by the most advanced techniques (including molecular, genetic, biochemical, etc. methods). The final diagnosis must be discussed at MDT sessions and any controversies must be put in the right context vis-á-vis clinical data, imaging information and surgical procedure. There is ample evidence to show how therapy may change the morphology (and grade) of brain tumours (Molnár and Berényi). Experience shows that morphological diagnoses are mostly reliable, problems usually arise when the above outlined theoretical and physical limits are neglected, or, in the worst case, if the pathologist is inexperienced (which unfortunately often is compensated by over-selfconfidence). Hence the verdict: every patient has the right for and is entitled to a second (third?) opinion, should the need arise! These requests must not ever be handled as "vanity" issues ${ }^{3}$. The wisdom of a pathologist is reflected by his humble (but not subservient) attitude firstly and outmost towards the patient. The question "Whose tissue is it, after all?" cannot be avoided either. Unfortunately with the dawn of tissue collection second opinions are often hindered by avaricious motivations of tissue banking and block-anxiety.

\subsection{Biomarkers in neuro-oncology}

In order to indentify the most adequat patient-tailored therapy the pathologist needs to identify 3 basic kinds of factors. These comprise diagnostic, prognostic and predictive markers. There is a standard but ever growing armamentarium of techniques for achieving these goals. Diagnostic markers are those which help to recognize those features which will allow the proper WHO classification of the lesion. Traditional histological stains, immunohistochemistry (IHC) and certain chromosomal and/or genetic characteristics belong to this category. It is mandatory to follow the current literature to realize that certain parameters may get moved from the list of one specific kind of markers to another. This is well exemplified by the chromosomal change commonly referred to as $1 \mathrm{p} 19 \mathrm{q}$ status which used to be a predictive marker, nowadays it is one of the most important diagnostic markers while having important prognostic significance as well. Prognostic markers are defined as such that allow formation of an opinion on the "outlook" of the patient. In other words, prognostic markers help to estimate progression free or overall survival of the patient. These again may be histological/cytological features (necrosis, proliferative potential) or genetic alterations. It has only recently been realized that mutation(s) of the cytoplasmic isocytratedehydrogenase enzyme, IDH-1/IDH-2 are of prognostic significance in gliomas. Predictive markers are those which help to define the most effective treatment for an individual brain

${ }^{3}$ This author has ample personal experience to the contrary! 
tumor and thus help to avoid the application of ineffective therapeutic interventions which would only induce unpleasant and dangerous complications without real therapeutical benefit. The most widely studied such marker is the methylation status of the promoter region of the MGMT gene (MGMT $=$ methylguanine-methyltransferase). Promoter methylation reduces the amount of the enzyme which is encoded by the gene and the smaller the amount of this repair enzyme the more effective are the alkylating drugs. Recently (BNOS meeting, Cambridge, July 1, 2011) the predictive value of the MGMT status has been seriously questioned, although it remains an important prognostic marker. It is thus important to note that there is an overlap between various members of these 3 groups of biological markers.

\section{Molecular and genetic background for biomarkers}

\subsection{Neuroepithelial tumors}

In hindsight it seems rightful to say that the work which started with the identification of altered clinical behavior of oligodendroglial tumors with $1 \mathrm{p} 19 \mathrm{q}$ co-deletions erupted in an unprecedented way once the study of 206 GBM's genome was published. One can confidently state that a new era in neuro-oncology started with the identification of the 3 major signalling pathways [RTK/RAS/PI(3)K (88\%), p53 (87\%) and Rb (78\%)]. Analysis of DNA copy numbers, gene-expression profiles and DNA-methylation patterns provided a whole new perspective for the proper evaluation of the real role of ERBB2, NF1 and TP53 genes. Frequent mutations of PIK3R1 have also been observed. The promoter methylation of the MGMT gene acquired unequivocal and clinically fundamental importance. It is now accepted that in treated cases of GBM the surfacing hypermutator phenotype is due to „mismatch repair deficiency".

Mutations may cause primer sequence-alterations, may be indicated by copy number changes and evidently may cause activation (gene amplification or overexpression) of oncogenes or inactivation (chromosomal loss or gene deletions) of tumor suppressor genes. The data published in Nature summarize the analysis of 97 million base pairs that is the somatic mutations of 601 genes. It became obvious that in GBMs with NF1 mutations RAF or MEK inhibitors may be effective, while CDK inhibitors may prove useful in tumors with CDKN2A or CDKN2C mutated GBMs.

Parsons et al published data on amplification and/or deletion patterns of 20.661 protein coding genes in 22 human GBMs. New methodologies (aCGH, high density oligonucleotide arrays, next generation sequencing technologies, single nucleotide genomics, massively parallel DNA resequencing) reconfirmed their most unexpected result: the earliest genetic change in most glial tumors affects the gene that encodes the active site of the cytoplasmic form of a carbohydrate-metabolizing enzyme, i. e., IDH = isocytrate dehydrogenase. The enzyme has several isoforms but now it is accepted that IDH-1 mutations are the most common in relatively young patients' secondary GBMs which have a better prognosis. The results have been confirmed by Blass et al, and Yan et al. and it has also been amply shown that IDH-1 mutations are highly specific for glial tumors, they do not occur in systemic forms of malignant diseases (certain types of AML are exceptions to the rule). Zhao et al published their observations in 2009 which showed that the mutations (IDH-1; R132 or IDH2; R172) reduce the enzyme's affinity towards its substrate plus inactive heterodimers appear which dominantly block the activity of the WT IDH-1 activity. There is a simultaneous induction of HIF-1a. A prime role was played in this work by Professor von 
Deimling and his co-workers in Heidelberg who not only showed (by direct genesequencing) that the IDH-1 mutation is missing from pilocytic astrocytomas but were also able to identify a BRAF-KIAAA1549 gene fusion which characterizes these tumors. Thus the Heidelberg group for the first time made it routinely possible to separate not only reactive astrocytes from neoplastic ones (reactive cells are IDH-1 negative with the commercially available antibody which they generated) but also solved the haunting problem of reliably identifying pilocytic astrocytomas.

It is not easy to portray this explosive evolution that has so basically changed the routine approach to glioma diagnostics. It has happened so rapidly that in a recent book on "NeuroOncology" (published by Elsevier as part of the "Blue Books of Neurology series in 2010) only one (yes, only 1) vague sentence is included on the importance of IDH-1 mutations. Meanwhile, in 2011, there is a general agreement amongst neuro-oncopathologists that a treatment-determining routine of glioma diagnostics should include the analysis of IDH-1 mutations (immunohistochemistry), checking of the $1 \mathrm{p} 19 \mathrm{q}$ status (FISH) and the mutations of TP53 (p53 IHC). These three steps are the minimum "must" for adequate diagnoses.

The following salient facts support the statement above. Except for the relatively rare primary GBMs it seems that the first step in glioma-genesis is the IDH-1 mutation. If that is followed by the damage of the $1 \mathrm{p} 19 \mathrm{q}$ regions then oligodendrogliomas or oligoastrocytic tumors develop. If contrarily, not the 1p19q loci are altered but the TP53 gene is mutated, then progression to secondary GBM is inevitable.

We currently think that primer (de novo) GBMs arise from a different progenitor (TIC) cell than all other gliomas, moreover, those genetic alterations which accumulate during the biological progression of the various neoplasms which belong to either group are also different. These differences obviously have an effect on the proper choice of tailored therapy protocols.

The intrinsic significance of IDH-1 mutations is explained by the fact that this cytoplasmic enzyme is a key element in lipid-synthesis, most likely is a regulator of stress-provoked protective mechanisms, definitely is an important factor in oxidative cellular mechanisms and as such functions as an important player in oxygen-sensor transduction signalling. The question whether it is or it is not 2HG (2-hydroxyglutarate; which is a metabolite of the IDH-catalyzed metabolic events) that interferes with cellular processes and thus induces neoplasms awaits further studies and eventual clarification.

It is truly amazing to experience the molecular revolution that has recently changed our concepts about glioma genesis. This process is in the making, as this chapter is being formulated. A very recent publication proves that despite rather similar phenotypic features anaplastic oligodendroglial tumors are biologically and clinically heterogeneous. NB. The problem of classifying and diagnosing oligodendroglial tumors solely based on cytomorphology or immunohistochemistry has been a problem that has haunted neurooncopathologists for a long time (just remember an elusive and highly subjective category: glioblastomas with a significant oligodendroglial component). Idbaih et al reported that thirty-three BACs (Bacterial Artificial Chromosome array) with prognostic value were identified which helped to separate 4 genomic subgroups of anaplastic oligodendroglial tumors (AOTs). Type I tumors (25\%) also displayed EGFR amplification, higher rate of necrosis and were associated with poor prognosis. Type II tumors were usually frontal tumors with the well known 1p19q loss and also had the usual oligodendroglial appearance. These patients $(21.7 \%)$ had a longer survival. Type III tumors $(11.7 \%)$ were characterized 
with deletion of prognostic BACs (21q) and had short survival. Type IV tumors are defined negatively by the absence of the genomic abnormalities seen in the other 3 groups and this represents $42 \%$ of all tumors. It is seriously thought-provoking that this material was comprised of samples of an EORTC trial, meaning, that all tumors were centrally reviewed and validated. What else could more convincingly illustrate the preceding paragraphs on analogous thinking and the issues related to subjectivity of pathological diagnoses? Regardless of some uncertainties associated with this series it is beyond doubt that soon we will see the overwhelming need of molecular sub-classification(s) of brain tumors which will be prerequisite for tailored therapy.

A similar tendency is present with regard to GBMs. Based on molecular analyses already in 2006 GBMs were sub-typed as mesenchymal and proneural. There was one group with a high number of CD133+ cells, with relatively sharp demarcation, cortical location. The lack of a high number of CD133+ cells was associated with a more expressed angiogeneic and proliferative activity. Verhaak et al continued the analysis and currently GBMs seem to belong to 4 subtypes: "classic", mesenchymal, proneural and neural. What is of outmost importance is that the canonical Stupp protocol seems to be effective only in the classic and mesenchymal subtypes. There is no further need to convince anyone that tailored, personalized, individual brain tumor therapy will very soon be more dependent upon molecular/genetic features than on traditional tinctorial and immunohistochemical profiles. Another recently recognized and increasingly significant observation concerns epigenetic features (hyper-, and hypomethylation) as well as the rapidly growing information on miRNAs' regulatory effects. Suffices to say though that the various molecular analyses cannot replace traditional morphology: the proper candidates for the adequate molecular tests will for a long time to come be chosen based on something like the WHO classification. This is demonstrated in Table 4. Entities are still being identified based on pattern recognition (i. e., traditional analogous thinking).

\begin{tabular}{|c|c|c|c|c|c|}
\hline \multirow[b]{2}{*}{ Glioma type } & \multicolumn{4}{|c|}{ Genomic abnormality } & \multirow[b]{2}{*}{$\begin{array}{l}\text { WHO } \\
\text { grade }\end{array}$} \\
\hline & $\begin{array}{l}\text { BRAF } \\
\text { alteration }\end{array}$ & $\begin{array}{c}1 \mathrm{p} 19 \mathrm{q} \\
\text { deletion }\end{array}$ & $\begin{array}{c}\text { IDH1 } \\
\text { mutation }\end{array}$ & $\begin{array}{c}\text { MGMT } \\
\text { methylation }\end{array}$ & \\
\hline $\begin{array}{l}\text { pilocytic } \\
\text { astrocytoma }\end{array}$ & + & neg & neg & neg & I \\
\hline oligodendroglioma & neg & + & + & + & II \\
\hline anaplastic ODG & neg & + & + & + & III \\
\hline $\begin{array}{l}\text { oligoastrocytoma } \\
\text { OA }\end{array}$ & neg & + & + & + & II \\
\hline anaplastic $\mathrm{OA}$ & neg & + & + & + & III \\
\hline $\begin{array}{l}\text { diffuse } \\
\text { astrocytoma }\end{array}$ & neg & neg & + & + & II \\
\hline $\begin{array}{l}\text { anaplastic } \\
\text { astrocytoma }\end{array}$ & neg & neg & + & + & III \\
\hline Secondary GBM & neg & neg & + & + & IV \\
\hline Primary GBM & neg & neg & + & + & IV \\
\hline
\end{tabular}

Table 4. 
Based upon the data in Table 4 it is obvious that state-of-the-art neuro-oncopathology is inconceivable without those analyses which are indicated in green. Lack of these results is highly counterproductive when it comes to tailored therapy and easily results in inadequate (or even harmful) chemotherapy.

It cannot be overemphasized that the various markers' definition and their clinical significance must from this time on serve as mental charts for neuro-oncologists, much more than the minute descriptions of morphological features. However, the latter are still of outmost importance for the pathologists, and these are easily accessible (a list of relevant reference books are found at the end of the chapter).

It is disappointing that ependymal tumors en masse show a much less well established list of easily detectable, well controlled, prospectively analyzed markers of either diagnostic and/or prognostic value. Recent data indicate that 3 groups of ependymomas might be possible to separate which do have significantly different survival rates. The best faring patients (Group 1; almost 100\% 5-year overall survival: OS) suffered from tumors which were characterized by gains of chromosome $9,15 \mathrm{q}$, and/or 18, plus loss of chromosome 6 . Group 2 patients with a 5-year OS around 70\% had mostly diploid genome. Group 3 patients harbored ependymomas with the worst prognosis (5-year OS less than 30\%) showed 1q gain and/or homozygous deletion of $9 \mathrm{p} 21$. It is important to note that often the actual chromosomal portion's function which is involved is not as yet clarified. Suffice it to say here that even for this highly elusive group of tumors there is light at the end of the tunnel and based on the recent past it can be expected that multicenter collaborative work on these entities will bring highly awaited results.

\subsection{Embryonal tumors}

Parallel to the evolution of molecular markers for gliomas a similar and fundamental change has occurred with regard to some embryonal tumors, namely, medulloblastomas. Similarly to those that apply to gliomas most of the required analyses are available for not only major centers but also for smaller laboratories, mainly due to the availability of immunohistochemical methods (i. e., proper and reliably tested antibodies: IDH-1, $\beta$-catenin, etc.)

Recent results indicate that medulloblastomas are highly heterogeneous and complex neoplasms, which require tailored treatment. Unfortunately on a daily basis therapeutic decisions for these patients are still predominantly made on risk group assignment based on clinical parameters and histological assessment takes place mainly of end stage disease. It is encouraging however, that our understanding of the molecular pathways involved in the pathogenesis of the majority of embryonal neoplasms is rapidly increasing. As in the case of systemic malignancies (breast-, lung-, colon cancer, and melanoma) molecular profiling of individual cancer reflects the individuality of each tumour, reveals the presence or absence of distinct disease-associated molecular signatures (see Table 4. and below). Hence these data represent a crucial component for the application of new molecular data obtained within the realm of basic oncology to tailored clinical practice.

Molecular subgroups in medulloblastomas (MBs) are characterized by distinct gene expression profiles. Convincing presentations at the British Neuro-oncology Society (Glasgow, 2010) and at the Salzburg Conference of the ISN (International Society of Neuropathology, 2010) plus several rather recent publications attest to the fact that activation of the WNT or SHH signalling pathway separates two such subgroups. The "WNT subgroup" (cca. 15\%) is associated with low-risk disease. New chemotherapeutics are 
available to target the $\mathrm{SHH}$ pathway (SHH subgroup: approximately 30\%). Active research is going on to further characterize the non-SHH/WNT type of medulloblastoma (more than $50 \%$ of MBs). Commercially available IHC reagents (GAB1, B-catenin, filamin A, and YAP1 antibodies) are helpful in distinguishing the various subtypes.

It is important to realize that MBs originate from cerebellar cortical progenitor cells. The first step seems to involve the activation of the WNT and Hedgehog (SHH) pathway. Activation of these two signalling pathways leads to the development of "classic" $\mathrm{MB}$, nodular/desmoplastic $\mathrm{MB}$ and medullomyoblastoma. Accumulation of further genetic abnormalities (c-myc and/or N-myc amplification/overexpression, 17p loss, hTERT amplification/overexpression, etc.) results in the phenotypic change that characterizes large cell/anaplastic MBs.

Ellison et al reported the IHC classification of medulloblastomas into 3 groups. Their modified table is presented below as Table 5 .

\begin{tabular}{|c|cccc|}
\multirow{2}{*}{ Pathway } & \multicolumn{4}{|c|}{ IHC decoration of } \\
& GAB1 & $\beta$-catenin & Filamin A & YAP1 \\
\cline { 1 - 2 } SHH & cytoplasmic $(\mathrm{CP})$ & $\mathrm{CP}$ & $\mathrm{CP}$ & nuclear + CP \\
\cline { 1 - 1 } WNT & negative & nuclear + CP & $\mathrm{CP}$ & nuclear + CP \\
\cline { 1 - 1 } non/SHH/WNT & negative & $\mathrm{CP}$ & negative & negative \\
\hline
\end{tabular}

Table 5.

Northcott et al have furthered this work and divided the non/SHH/WNT group into "Group C" and "Group D". Their exon array analysis was supplemented with IHC detection of the related proteins. The differential expression of 4 genes (DKK1, SFRP1, NPR3, and KCNA1) seems to correlate with variability of age groups and risk categories. IHC comprised antibodies against $\beta$-catenin, DKK1, SFRP1, GLI1, and KCNA1 proteins. The two papers were published during a 2-months-long period within the last six months. This only goes to show how rapidly this field is changing. It is to be tested on an ever increasing number of patients with proper stratification and statistical analysis but it is already clear that at least part of the late and unfortunate consequences of radical chemo- and radiotherapy of MBs can be avoided.

\subsection{Meningiomas}

Loss of 22q has long been described in meningeal tumors, however, no prognostic value has been possible to link to this alteration which occurs in about $60 \%$ of all meningiomas. It is important that $1 p$ and $14 q$ deletions are of diagnostic value when the not infrequent issue of separating meningiomas from hemangiopericytomas, superficial GBMs or metastatic carcinomas is at stake. It is also documented that allelic losses on $1 \mathrm{p}$ are characteristic features of meningiomas with a higher grade. Probably the most important observation is that loss of $14 \mathrm{q}$ seems to correlate with not only higher grade but also with an increased frequency of recurrence. Our results (Molnár et al. to be published) indicate that ploidy measurements combined with nuclear digitized morphometry helps to divide meningiomas 
basically into two major groups with significantly different tendency to recur or progress towards higher grades.

\subsection{Primary CNS lymphomas, PCNSLs}

Statistically the dominant forms are B-cell derived neoplasms. Determination of the proper treatment is fundamentally dependent on the results of various IHC reactions. It is notable that these data are of both diagnostic-, prognostic- and predictive value. Separation of PCNSLs into germinal center type (GC) or postGC/nonGC subtypes has become the standard procedure. The most widely used IHC panel is comprised of CD10, CD20, Bcl-6, IRF4/MUM1 antibodies. Details of data interpretation are discussed adequately in various handbooks/textbooks on the neoplasms of the hematopoietic-lymphoid tissues.

It is to be kept in mind that the distinctive capillary types of brain tumours (BTB = bloodtumour barrier [Schlageter et al]) have a unique feature in PCNLs: endothelial cells tend to undergo apoptosis as a result of steroid treatment (Molnár et al).

\subsection{Metastatic tumors}

An often encountered problem is that systemic cancers often present themselves as brain or less frequently - spinal cord metastases. The thorough discussion of all problems related to clarifying the exact origin of MCUPs (metastatic cancer of unknown primary) exceeds the limits of this chapter.

\section{Concluding remarks}

1. Traditional histotechniques continue to provide the basis for starting to evaluate neurosurgical samples of neoplastic disease of the CNS.

2. If the lesion is a glial tumor IDH-1/2 analysis is to be complemented with the determination of the $1 \mathrm{p} 19 \mathrm{q}$ status and IHC analysis of p53.

3. If the tumor's glial nature is equivocal IDH-1/2 IHC should be decisive and needs to be complemented by EGFR amplification analysis. If EGFR amplification is present with IDH1 mutation then diagnosis of GBM is warranted.

4. Grade determination might be supported and validated by IDH-1/2 analysis, $1 \mathrm{p} 19 \mathrm{q}$, EGFR and p16 FISH. MGMT methylation status is crucial in deciding the actual use of already widely available chemotherapeutic protocols (e. g., Stupp's).

5. Medulloblastomas already are feasible to stratification into low and high risk groups.

6. Primary CNS lymphomas can be grouped as B or $\mathrm{T}$ cell neoplasms and the most common type (DLBCL = diffuse large B cell lymphoma) can be separated into GC (Germinal Center) types and post GC types with the conventional IHC panels.

7. Metastatic lesions should be worked up along the lines which are well established in systemic pathology.

Recently, techniques such as next-generation DNA sequencing, massively parallel DNA resequencing and 'single-molecule genomics' have revolutionized cancer genomics. The results are "pouring in" and we can all expect that these will make a huge impact on personalized medicine in the near future. It definitely is an exceedingly exciting time ahead and at last neuro-oncopathologists may have positive intellectual reinforcements when trying to integrate this information into a morpho-functional interpretation. 


\section{Acknowledgement}

The author is grateful for the support of the National Cancer Center, New York, NY (USA) and the VFK Krebsforschung GmbH. (Germany). Research Grant \# 516/2010, Dr. Molnár P.

\section{References}

AJCC Cancer Staging Handbook. From the AJCC Cancer Staging Manual. Edge SB, Byrd DR, Compton CC, Fritz AG, Greene FL, Trotti A. (Eds.) 7th Ed. Springer, New York/Dordrecht/Heidelberg, London. 2010. pp 651-660.

Bailey P, Cushing H: A Classification of the Tumors of the Glioma Group on a Histogenetic Basis with a Correlated Study of Prognosis. Philadelphia: Lippincott, 1926.

Balss J, Meyer J et al: Analysis of the IDH1 codon 132 mutation in brain tumors. Acta Neuropath 116: 597-602, 2008.

Burger PC, Scheithauer BW et al: Surgical Pathology of the Nervous System and Its Coverings. 4th ed. Churchill Livingstone, Philadelphia. 2002.

Carlsson A, Persson $\mathrm{O}$ et al: Plasma proteome profiling reveals biomarker patterns associated with prognosis and therapy selection in glioblastoma multiforme patients. Proteomics Clin Appl 4: 591-602, 2010. doi:101002/prca.200900173

CBTRUS. Annual report. Chicago: Central Brain Tumor Registry of the United States, 2010.

Garber K. Oncometabolite? IDH1 discoveries raise possibility of new metabolism targets in brain cancers and leukaemia. JNCI 102: 926-928, 2010.

Goutagny S, Yang HW et al: Genomic Profiling Reveals Alternative Genetic Pathways of Meningioma Malignant Progression Dependent on the Underlying NF2 Status. Clin Cancer Res 16:4155-4164, 2010

Gozé C, Rigau V et al: Lack of complete 1p19q deletion in a consecutive series of 12 WHO grade II gliomas involving the insula: a marker of worse prognosis? J Neuroncol 91: 1-5, 2009. doi:10.1007/s11060-008-9680-8

Huse JT and Holland EC. Targeting brain cancer: advances in the molecular pathology of malignant glioma and medulloblastoma. Nature Reviews Cancer 10: 319-331, 2010. doi:10.1038/nrc2818

Jakab A, Molnár P et al: Glioma grade assessment by using histogram analysis of diffusion tensor imaging-derived maps. Neuroradiology 2010. doi:10.1007/s00234-010-07693)

Jeuken JWM, van der Maazen, Wesseling P. Molecular diagnostics as a tool to personalize treatments in adult glioma patients. Technology in Canc Res and Treatment 5: 1-15, 2008.

Joo $\mathrm{MH}$ et al. Clinical and biological implications of CD133-positive and CD133-negative cells in glioblastomas. Lab Invest 88: 808-815, 2008.

Kernohan JW, Mabon RF et al: A simplified classification of the gliomas. Mayo Clin Proc 24: 71-75, 1949. 
Korshunov A, Meyer J et al: Combined molecular analysis of BRAF and IDH1 distinguishes pilocytic astrocytoma from diffuse astrocytoma. Acta Neuropathol 118: 401-405, 2009. doi:10.1007/s00401-009-0550-z

Korshunov A, Witt $\mathrm{H}$ et al: Molecular staging of intracranial ependymoma in children and adults. J Clin Oncol 28: 1-14, 2010. doi:10.1200/JCO.2009.27.3359

Loius DN, Ohgaki H et al: The 2007 WHO Classification of Tumours of the Central Nervous System. Acta Neuropathol 114: 97-109, 2007. doi:10.1007/s00401-007-0243-4

Mawrin $\mathrm{Ch}$ and Perry A. Pathological classification and molecular genetics of meningiomas. J Neuro-Oncol. Published online Sept. 01, 2010. doi:10.1007/s11060-010-0342-2

Molnár PP, Berényi E. Therapy-induced morphological alterations in brain tumors. The Open Pathology Journal 3: 81-90, 2009

Molnár PP. O'Neill BP et al: The blood-brain barrier in primary CNS lymphomas: Ultrastructural evidence of endothelial cell death. Neuro-Oncology 1: 89-100, 1998.

Ohgaki $\mathrm{H}$ and Kleihues P. Genetic alterations and signalling pathways in the evolution of gliomas. Cancer Science 100: 2235-2241, 2009.

Parsons DW et al. An Integrated Genomic Analysis of Human Glioblastoma Multiforme. Science 321: 1807-1812, 2008.

Perry A and Brat DJ. Practical Surgical Neuropathology. A Diagnostic Approach. Churchill Livingstone/Elsevier. Philadelphia. 2010

Philips HS et al. Molecular subclasses of high-grade glioma predict prognosis, delineate a pattern of disease progression, and resemble stages of neurogenesis. Cancer Cell 9: 157-173, 2006.

Rivera AL and Pelloski ChE. Diagnostic and prognostic molecular markers in common adult gliomas. Expert Rev. Mol Diagn 10: 637-649, 2010.

Schlageter KE, Molnar P et al: Microvessel organization and structure in experimental brain tumors: microvessel populations with distinctive structural and functional properties. Microvasc Res 58: 312-328, 1999

Shete $\mathrm{S}$ et al. Genome-wide association study identifies five susceptibility loci for glioma. Nature Gen 41: 899-904, 2009. doi:10.1038/ng.407

Stupp R, Mason WP, et al: Radiotherapy plus concomitant and adjuvant temozolomide for glioblastoma. N Engl J Med. 352: 987-96, 2005.

The Cancer Genome Atlas Research Network. Comprehensive genomic characterization defines human glioblastoma genes and core pathways. Nature 455: 1061-1068, 2008. doi:10.1038/nature07385

UICC (Union for International Cancer Control) http://www.uicc.org Sobin L, Gospodarowicz M, Wittekind Ch. TNM Classification of Malignant Tumours. 7th Ed. UICC. Wiley-Blackwell, Oxford. 2010.

Verhaak RGV et al. Integrated Genomic Analysis Identifies Clinically Relevant Subtypes of Glioblastoma Characterized by Abnormalities in PDGFRA, IDH1, EGFR, and NF1 Cancer Cell 17: 98-110, 2010.

von Deimling A. British Neuro-Oncological Society, BNOS Conference. Glasgow. 2010. 
WHO Classification of Tumours of Haematopoietic and Lymphoid tissues. WHO/IARC. Swerdlow SH, Campo E et al, Lyon, 2008. pp 20-241.

WHO Classification of Tumours of the Central Nervous System. Louis DN, Ohgaki H et al: International Agency for Research on Cancer (IARC). Lyon. 2007.

Wibom C, Mörén L et al: Proteomic profiles differ between bone invasive and noninvasive benign meningiomas of fibrous and meningothelial subtype. J Neuro-Oncol 94: 321-331, 2009.

Yan $\mathrm{H}$ et al. IDH1 and IDH2 Mutations in Gliomas. NEJM 360: 13-21, 2009.

Yan H, Bigner DD, et al: Mutant metabolic enzymes are at the origin of gliomas. Cancer Res 69: 9157-9159, 2009. doi:10.1158/0008-5472.CAN-09-2650

Zachary JR and Yan H. Isocitrate dehydrogenase 1 and 2 mutations in cancer: alterations at a crossroads of cellular metabolism. JNCI 102: 932-941, 2010.

Zhao Sh et al. Glioma-Derived Mutations in IDH1 Dominantly Inhibit IDH1 Catalytic Activity and Induce HIF-1a. Science 324: 261-265, 2009. 


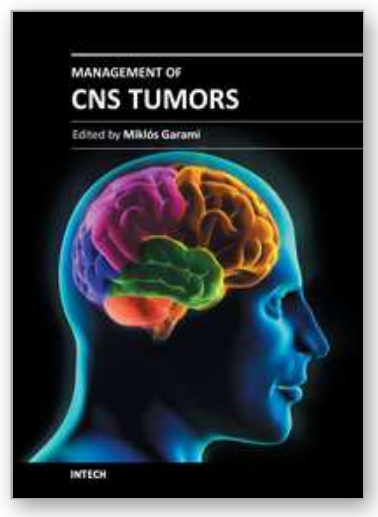

\author{
Management of CNS Tumors \\ Edited by Dr. Miklos Garami
}

ISBN 978-953-307-646-1

Hard cover, 464 pages

Publisher InTech

Published online 22, September, 2011

Published in print edition September, 2011

Management of CNS Tumors is a selected review of Central Nervous System (CNS) tumors with particular emphasis on pathological classification and complex treatment algorithms for each common tumor type. Additional detailed information is provided on selected CNS tumor associated disorders.

\title{
How to reference
}

In order to correctly reference this scholarly work, feel free to copy and paste the following:

Péter Molnár (2011). Classification of Primary Brain Tumors: Molecular Aspects, Management of CNS Tumors, Dr. Miklos Garami (Ed.), ISBN: 978-953-307-646-1, InTech, Available from:

http://www.intechopen.com/books/management-of-cns-tumors/classification-of-primary-brain-tumorsmolecular-aspects

\section{INTECH}

open science | open minds

\section{InTech Europe}

University Campus STeP Ri

Slavka Krautzeka 83/A

51000 Rijeka, Croatia

Phone: +385 (51) 770447

Fax: +385 (51) 686166

www.intechopen.com

\section{InTech China}

Unit 405, Office Block, Hotel Equatorial Shanghai

No.65, Yan An Road (West), Shanghai, 200040, China

中国上海市延安西路 65 号上海国际贵都大饭店办公楼 405 单元

Phone: +86-21-62489820

Fax: $+86-21-62489821$ 
(C) 2011 The Author(s). Licensee IntechOpen. This chapter is distributed under the terms of the Creative Commons Attribution-NonCommercialShareAlike-3.0 License, which permits use, distribution and reproduction for non-commercial purposes, provided the original is properly cited and derivative works building on this content are distributed under the same license. 